\title{
Dissemination of data measured at the CERN n_TOF facility
}

E. Dupont ${ }^{1, a}$, N. Otuka ${ }^{2}$, O. Cabellos ${ }^{3}$, O. Aberle ${ }^{4}$, G. Aerts ${ }^{1}$, S. Altstadt ${ }^{5}$, H. Alvarez ${ }^{6}$, F. Alvarez-Velarde ${ }^{7}$, S. Andriamonje ${ }^{1}$, J. Andrzejewski ${ }^{8}$, L. Audouin ${ }^{9}$, M. Bacak ${ }^{4,10,1}$, G. Badurek ${ }^{10}$, J. Balibrea ${ }^{7}$, M. Barbagallo ${ }^{11}$, S. Barros $^{12}$, P. Baumann ${ }^{13}$, V. Bécares ${ }^{7}$, F. Bečvár ${ }^{14}$, C. Beinrucker ${ }^{5}$, F. Belloni ${ }^{15}$, B. Berthier ${ }^{9}$, E. Berthoumieux ${ }^{1}$, J. Billowes ${ }^{16}$, V. Boccone ${ }^{4}$, D. Bosnar ${ }^{17}$, A. Brown ${ }^{18}$, M. Brugger ${ }^{4}$, M. Caamaño ${ }^{6}$, M. Calviani ${ }^{4}$, F. Calviño ${ }^{19}$, D. Cano-Ott ${ }^{7}$, R. Capote $^{2}$, R. Cardella ${ }^{4}$, C. Carrapiço ${ }^{12}$, A. Casanovas ${ }^{19}$, D.M. Castelluccio ${ }^{20}$, P. Cennini ${ }^{4}$, F. Cerutti ${ }^{4}$, Y.H. Chen ${ }^{9}$, E. Chiaveri ${ }^{4}$, M. Chin $^{4}$, N. Colonna ${ }^{11}$, G. Cortés ${ }^{19}$, M.A. Cortés-Giraldo ${ }^{21}$, L. Cosentino ${ }^{22}$, A. Couture ${ }^{23}$, J. Cox ${ }^{23}$, L.A. Damone ${ }^{11}$, S. David ${ }^{9}$ K. Deo ${ }^{24}$, M. Diakaki ${ }^{1}$, I. Dillmann ${ }^{25}$, C. Domingo-Pardo ${ }^{26}$, R. Dressler ${ }^{27}$, W. Dridi ${ }^{1}$, I. Duran ${ }^{6}$, C. Eleftheriadis ${ }^{28}$, M. Embid-Segura ${ }^{7}$, B. Fernández-Domínguez ${ }^{6}$, L. Ferrant ${ }^{9}$, A. Ferrari ${ }^{4}$, P. Ferreira ${ }^{12}$, P. Finocchiaro ${ }^{22}$, K. Fraval ${ }^{1}$, R.J.W. Frost ${ }^{16}$, K. Fujii ${ }^{15}$,W. Furman $^{29}$, S. Ganesan ${ }^{24}$, A.R. Garcia ${ }^{7}$, A. Gawlik ${ }^{8}$, I. Gheorghe ${ }^{30}$, S. Gilardoni ${ }^{4}$, G. Giubrone ${ }^{26}$, T. Glodariu ${ }^{30}$, K. Göbel ${ }^{5}$, M.B. Gomez-Hornillos ${ }^{19}$, I.F. Goncalves ${ }^{12}$, E. Gonzalez-Romero ${ }^{7}$, A. Goverdovski ${ }^{31}$, F. Gramegna ${ }^{32}$, E. Griesmayer ${ }^{10}$, C. Guerrero ${ }^{21}$, F. Gunsing ${ }^{1,4}$, P. Gurusamy ${ }^{24}$, R. Haight ${ }^{33}$, H. Harada ${ }^{34}$, T. Heftrich ${ }^{5}$, M. Heil ${ }^{25}$, S. Heinitz ${ }^{27}$, A. Hernández-Prieto ${ }^{19}$, J. Heyse ${ }^{35}$, M. Igashira ${ }^{36}$, S. Isaev ${ }^{9}$, D.G. Jenkins ${ }^{18}$, E. Jericha ${ }^{10}$, Y. Kadi ${ }^{4}$, F. Kaeppeler ${ }^{25}$, A. Kalamara ${ }^{37}$, D. Karadimos ${ }^{38}$, D. Karamanis ${ }^{38}$, T. Katabuchi ${ }^{36}$, P. Kavrigin ${ }^{10}$, M. Kerveno ${ }^{13}$, V. Ketlerov ${ }^{31}$, V. Khryachkov ${ }^{31}$, A. Kimura ${ }^{34}$, N. Kivel ${ }^{27}$, M. Kokkoris ${ }^{37}$, V. Konovalov ${ }^{31}$, M. Krtička ${ }^{14}$, J. Kroll ${ }^{14}$, D. Kurtulgil ${ }^{5}$, C. Lampoudis ${ }^{28}$, C. Langer $^{5}$, E. Leal-Cidoncha ${ }^{6}$, C. Lederer ${ }^{39}$, H. Leeb ${ }^{10}$, C. Le Naour ${ }^{9}$, J. Lerendegui-Marco ${ }^{21}$, L.S. Leong 9 , M. Licata ${ }^{40}$, S. Lo Meo ${ }^{20}$, S.J. Lonsdale ${ }^{39}$, R. Losito ${ }^{4}$, M. Lozano ${ }^{21}$, D. Macina ${ }^{4}$, A. Manousos ${ }^{28}$, J. Marganiec ${ }^{8}$, T. Martinez ${ }^{7}$, S. Marrone ${ }^{11}$, A. Masi ${ }^{4}$, C. Massimi ${ }^{40}$, P. Mastinu ${ }^{32}$, M. Mastromarco ${ }^{11}$, F. Matteucci ${ }^{15}$, E.A. Maugeri ${ }^{27}$, A. Mazzone ${ }^{11}$, E. Mendoza ${ }^{7}$, A. Mengoni ${ }^{20}$, P.M. Milazzo ${ }^{15}$, F. Mingrone ${ }^{40,4}$, M. Mirea ${ }^{30}$, W. Mondelaers ${ }^{35}$, S. Montesano ${ }^{4}$, C. Moreau ${ }^{15}$, M. Mosconi2 ${ }^{25}$, A. Musumarra ${ }^{41}$, A. Negret ${ }^{30}$, R. Nolte ${ }^{42}$, S. O’Brien ${ }^{23}$, A. Oprea ${ }^{30}$, F.R. Palomo-Pinto ${ }^{21}$, J. Pancin ${ }^{1}$, C. Paradela ${ }^{6,35}$, N. Patronis ${ }^{38}$, A. Pavlik ${ }^{43}$, P. Pavlopoulos ${ }^{4}$, J. Perkowski ${ }^{8}$, L. Perrot ${ }^{1}$, M.T. Pigni ${ }^{10}$, R. Plag 25 , A. Plompen ${ }^{35}$, L. Plukis ${ }^{1}$, A. Poch ${ }^{44}$, I. Porras ${ }^{45}$, J. Praena ${ }^{45}$, C. Pretel ${ }^{44}$, J.M. Quesada ${ }^{21}$, D. Radeck ${ }^{42}$, K. Rajeev ${ }^{24}$, T. Rauscher ${ }^{46,47}$, R. Reifarth ${ }^{5}$, A. Riego ${ }^{19}$, M. Robles ${ }^{6}$, F. Roman ${ }^{4}$, P.C. Rout ${ }^{24}$, G. Rudolf ${ }^{13}$, C. Rubbia ${ }^{4}$, P. Rullhusen ${ }^{35}$, J.A. Ryan ${ }^{16}$, M. Sabaté-Gilarte ${ }^{4}$, J. Salgado ${ }^{12}$, C. Santos ${ }^{12}$, L. Sarchiapone ${ }^{4}$, R. Sarmento ${ }^{12}$, A. Saxena ${ }^{24}$, P. Schillebeeckx ${ }^{35}$, S. Schmidt ${ }^{5}$, D. Schumann ${ }^{27}$, P. Sedyshev ${ }^{29}$, A.G. Smith ${ }^{16}$, N.V. Sosnin ${ }^{16}$, A. Stamatopoulos ${ }^{37}$, C. Stephan ${ }^{9}$, S.V. Suryanarayana ${ }^{24}$, G. Tagliente ${ }^{11}$, J.L. Tain ${ }^{26}$,

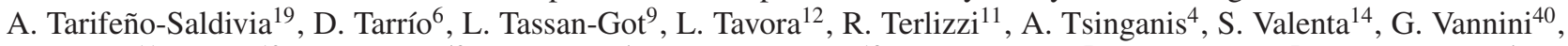
V. Variale ${ }^{11}$, P. Vaz ${ }^{12}$, A. Ventura ${ }^{40}$, R. Versaci ${ }^{4}$, M.J. Vermeulen ${ }^{18}$, D. Villamarin ${ }^{7}$, M.C. Vicente ${ }^{7}$, V. Vlachoudis ${ }^{4}$,

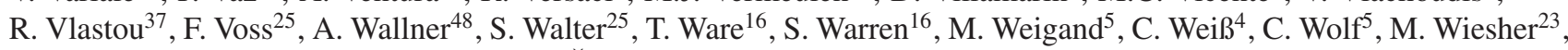
K. Wisshak ${ }^{25}$, P.J. Woods ${ }^{39}$, T. Wright ${ }^{16}$, P. Žugec ${ }^{17}$, and the n_TOF Collaboration (www.cern.ch/ntof)

1 CEA, Irfu, Universiteí Paris-Saclay, Gif-sur-Yvette, France

2 IAEA Nuclear Data Section, Vienna, Austria

OECD Nuclear Energy Agency, Boulogne-Billancourt, France

${ }^{4}$ CERN, European Organization for Nuclear Research, Geneva, Switzerland

5 Johann-Wolfgang-Goethe Universität, Frankfurt, Germany

${ }^{6}$ Universidade de Santiago de Compostela, Spain

7 CIEMAT, Centro de Investigaciones Energeticas Medioambientales y Tecnológicas, Madrid, Spain

8 University of Lodz, Poland

9 IPN, CNRS-IN2P3, Université Paris-Sud, Université Paris-Saclay, Orsay, France

10 TUW, Technische Universität Wien, Vienna, Austria

11 INFN, Istituto Nazionale di Fisica Nucleare, Bari, Italy

12 Instituto Superior Técnico, Universidade de Lisboa, Lisbon, Portugal

13 CNRS/IN2P3 - IPHC, Strasbourg, France

14 Charles University, Prague, Czech Republic

15 INFN, Istituto Nazionale di Fisica Nucleare, Trieste, Italy

16 University of Manchester, UK

17 Department of Physics, Faculty of Science, University of Zagreb, Croatia

18 University of York, UK

a e-mail: emmeric.dupont@cea.fr 
19 Universitat Politécnica de Catalunya, Barcelona, Spain

20 ENEA, Agenzia nazionale per le nuove tecnologie, Bologna, Italy

21 Universidad de Sevilla, Spain

${ }^{22}$ INFN - Laboratori Nazionali del Sud, Catania, Italy

23 University of Notre Dame, Indiana, USA

${ }^{24}$ BARC, Bhabha Atomic Research Centre, Mumbai, India

25 KIT, Karlsruhe Institute of Technology, Karlsruhe, Germany

26 Instituto de Física Corpuscular, Universidad de Valencia, Spain

27 PSI, Paul Scherrer Institut, Villigen, Switzerland

28 Aristotle University of Thessaloniki, Greece

29 JINR, Dubna, Russia

${ }^{30}$ Horia Hulubei National Institute of Physics and Nuclear Engineering, Bucharest - Magurele, Romania

31 IPPE, Obninsk, Russia

32 INFN - Laboratori Nazionali di Legnaro, Italy

33 LANL, New Mexico, USA

34 JAEA, Japan Atomic Energy Agency, Japan

35 EC-JRC-Geel, European Commission, Joint Research Centre, Geel, Belgium

36 Tokyo Institute of Technology, Japan

37 NTUA, National Technical University of Athens, Greece

38 University of Ioannina, Greece

39 University of Edinburgh, UK

40 Dipartimento di Fisica, Università di Bologna, and Sezione INFN di Bologna, Italy

41 Dipartimento di Fisica e Astronomia, Università di Catania and INFN-Laboratori Nazionali del Sud, Catania, Italy

42 PTB, Physikalisch-Technische Bundesanstalt, Braunschweig, Germany

43 University of Vienna, Faculty of Physics, Vienna, Austria

44 Universidad Politécnica de Madrid, Spain

45 Universidad de Granada, Spain

46 Centre for Astrophysics Research, University of Hertfordshire, UK

47 Department of Physics, University of Basel, Switzerland

48 Australian National University, Canberra, Australia

\begin{abstract}
The n_TOF neutron time-of-flight facility at CERN is used for high quality nuclear data measurements from thermal energy up to hundreds of $\mathrm{MeV}$. In line with the CERN open data policy, the n_TOF Collaboration takes actions to preserve its unique data, facilitate access to them in standardised format, and allow their re-use by a wide community in the fields of nuclear physics, nuclear astrophysics and various nuclear technologies. The present contribution briefly describes the $\mathrm{n}_{-}$TOF outcomes, as well as the status of dissemination and preservation of $n_{-}$TOF final data in the international EXFOR library.
\end{abstract}

\section{Introduction}

The CERN n_TOF facility [1] is used since 2001 for high quality nuclear data measurements. A considerable amount of valuable experimental results have been obtained and published, and new measurements are going on. These results are important for the end-users of nuclear data, in particular for the improvement of evaluated nuclear data libraries. In line with global standards in data preservation and Open Science, the $\mathrm{n}_{-} \mathrm{TOF}$ Collaboration takes actions to preserve its unique data, facilitate access to them in standardised format, and allow their re-use by a wide community in the fields of nuclear physics, nuclear astrophysics and various nuclear technologies for medical and energy applications. In the latter fields, all evaluated nuclear reaction libraries are built from the international EXFOR library [2], which has been successfully developed and maintained for 50 years by the International Network of Nuclear Reaction Data Centres (NRDC). Accordingly, the n_TOF Collaboration strengthened the links with the IAEA Nuclear Data Section and the NEA Data Bank to improve the dissemination and preservation of its data through the EXFOR library. The present paper describes the main $\mathrm{n}_{-} \mathrm{TOF}$ outcomes, and the status of data dissemination activities.

\section{The n_TOF outcomes}

The facility is based on the $20 \mathrm{GeV} / \mathrm{c}$ pulsed proton beam from CERN's Proton Synchrotron impinging on a lead spallation target. A layer of water around the spallation target moderates the initially fast neutrons down to a white spectrum of neutrons covering the full range between $\mathrm{meV}$ and $\mathrm{GeV}$ neutron energies.

During Phase-I when the first spallation target was used from 2001 up to 2004, the water coolant also served as moderator. Starting from Phase-II, after the installation in 2008 of an upgraded spallation target, the latter was enclosed with a separate cooling circuit followed by an exchangeable borated water moderator.

Since 2001, neutron induced reactions are measured in the experimental area EAR-1 [3] at approximately $185 \mathrm{~m}$ from the spallation source. In 2014, a second experimental area, EAR-2 [4], at about $18 \mathrm{~m}$ from the neutron source has been constructed for Phase-III and is offering new possibilities for demanding cross section measurements. Table 1 gives an overview of the number of measurements since 2001.

\subsection{Phase-I (2001-2004) \& Phase-II (2009-2012)}

During the first phase from 2001 to 2004 capture and fission data for a number of isotopes have been 
taken. Capture measurements with $\mathrm{C}_{6} \mathrm{D}_{6}$ liquid scintillator detectors concerned both stable and radioactive isotopes of $\mathrm{Mg}, \mathrm{Fe}, \mathrm{Zr}, \mathrm{La}, \mathrm{Sm}, \mathrm{Os}, \mathrm{Au}, \mathrm{Pb}, \mathrm{Bi}$, and Th. A $4 \pi$ Total Absorption Calorimeter (TAC) [5] consisting of $40 \mathrm{BaF}_{2}$ crystals has been used for neutron capture measurements of ${ }^{197} \mathrm{Au},{ }^{233} \mathrm{U},{ }^{234} \mathrm{U},{ }^{237} \mathrm{~Np},{ }^{240} \mathrm{Pu}$, and ${ }^{243} \mathrm{Am}$. Fission cross sections were measured with the FIC fission detectors [6] for ${ }^{232} \mathrm{Th},{ }^{233} \mathrm{U},{ }^{234} \mathrm{U},{ }^{235} \mathrm{U},{ }^{236} \mathrm{U},{ }^{238} \mathrm{U}$, ${ }^{237} \mathrm{~Np},{ }^{241} \mathrm{Am},{ }^{243} \mathrm{Am}$, and ${ }^{245} \mathrm{Cm}$. Other fission detectors based on Parallel Plate Avalanche Counters (PPAC) [7,8] were used in measurements of the fission cross sections of ${ }^{n a t} \mathrm{~Pb},{ }^{209} \mathrm{Bi},{ }^{232} \mathrm{Th},{ }^{237} \mathrm{~Np},{ }^{233} \mathrm{U},{ }^{234} \mathrm{U},{ }^{235} \mathrm{U}$ and ${ }^{238} \mathrm{U}$. The full list of measured isotopes and reactions together with the final or most relevant publication is given in Ref. [9].

During Phase-II in 2009-2012 mostly capture measurements were performed, while new PPAC were used for additional fission experiments, including the measurement of the fission-fragment angular distributions [10]. The $(\mathrm{n}, \gamma)$ reaction was investigated on additional stable and radioactive isotopes of $\mathrm{Mg}, \mathrm{Fe}, \mathrm{Ni}$, and $\mathrm{Zr}$. In order to handle unsealed, but still encapsulated, highly radioactive samples, EAR-1 was transformed in a type A laboratory. After this transformation, measurements of the capture reactions were performed for ${ }^{236} \mathrm{U},{ }^{238} \mathrm{U}$ and ${ }^{241} \mathrm{Am}$, for the latter two with two different capture detector systems $\left(\mathrm{C}_{6} \mathrm{D}_{6}\right.$ and TAC). The TAC was also used in combination with a Micromegas detector in a first attempt to measure the ${ }^{235} \mathrm{U}(\mathrm{n}, \gamma)$ reaction using a veto on the fission reaction. In addition to these measurements several other techniques have been tested and the full list of the Phase-II measurements and their references are given in Ref. [9].

Table 1. Overview of the number of $\mathrm{n}_{-}$TOF measurements.

\begin{tabular}{|l|c|c|c|}
\hline Reaction & $\begin{array}{c}\text { Phase-I } \\
(\mathbf{2 0 0 1 - 2 0 0 4 )}\end{array}$ & $\begin{array}{c}\text { Phase-II } \\
(\mathbf{2 0 0 9}-\mathbf{2 0 1 2})\end{array}$ & $\begin{array}{c}\text { Phase-III } \\
(\mathbf{2 0 1 4}-. . .)^{*}\end{array}$ \\
\hline$(\mathbf{n}, \boldsymbol{\gamma})$ & 27 & 16 & $12(+14)$ \\
\hline$(\mathbf{n}, \mathbf{f})$ & 18 & 4 & $6(+2)$ \\
\hline (n,cp) & 0 & 3 & $5(+1)$ \\
\hline
\end{tabular}

*The numbers between parentheses correspond to additional approved measurements, as of March 2017.

\subsection{Phase-III (2014-...)}

Since 2014, two experimental areas are available for capture and fission measurements, and new challenging experiments are now possible in EAR-2, such as ${ }^{240} \mathrm{Pu}(\mathrm{n}, \mathrm{f})$, ${ }^{244} \mathrm{Cm}(\mathrm{n}, \gamma),{ }^{246} \mathrm{Cm}(\mathrm{n}, \gamma),{ }^{7} \mathrm{Be}(\mathrm{n}, \alpha)$ and ${ }^{7} \mathrm{Be}(\mathrm{n}, \mathrm{p})$. The list of the 2014-2015 measurements is available in Ref. [9] and an update is given in Ref. [11].

\section{Data dissemination}

Publication of results is obviously essential for knowledge transfer and preservation, but this is not enough when dealing with nuclear data for applications. Indeed, these data should be usable and actually used. Hence, once the data are finalized, published and fully exploited by the n_TOF Collaboration, it is essential to make them widely available to all users.

In the field of nuclear technologies especially, the EXFOR library is known as the "Mother of all libraries" and is the ground for the development of all evaluated nuclear reaction libraries for particle transport, activation, dosimetry, standards, etc. Moreover, EXFOR has been successfully maintained for decades and is continuously updated by the NRDC network. These two reasons make EXFOR an appropriate place for both an efficient dissemination and preservation of $\mathrm{n}_{-} \mathrm{TOF}$ data.

\subsection{IAEA recommendations}

The IAEA organised in 2013 a meeting on EXFOR data in the resonance region and spectrometer response functions [12]. Participants agreed on recommendations and prepared templates for submission of time-offlight spectra to NRDC. The main recommendations for experimentalists can be summarized as follows.

- Reaction yield or transmission should be reported in addition to cross sections.

- Data should be reported vs. time-of-flight in addition to the required neutron energy.

- Total uncertainty and uncorrelated uncertainty components should be reported separately.

- Additional information necessary to reconstruct the full covariance matrix should be reported if correlations due to data reduction are significant.

- Additional quantities of interest should be reported, such as the neutron flux distribution, energydependent backgrounds and correction factors, as well as the spectrometer response function to be used with the data.

\subsection{Dissemination of $n_{-}$TOF final data}

The raw $n_{-}$TOF data are available on CERN computers, whereas the final reduced data of interest to users are generally only available from the physicists doing the analysis. In order to release and preserve these final data, the $\mathrm{n}_{-}$TOF authors use the IAEA recommendations and templates as guidelines for the information to be supplied, with emphasis on data not already available in the publications.

In order to quantify the status and progress of the dissemination of $\mathrm{n}_{-} \mathrm{TOF}$ data, one should distinguish between various acceptable levels of completeness in data reporting. After publication of the final results, it is acknowledged that the necessary data to disseminate and preserve are the main experimental quantities (such as pointwise reaction yields, cross sections, crosssection ratios) and the associated statistical/uncorrelated uncertainty $v s$. energy with the best possible resolution. The status of $\mathrm{n}_{-} \mathrm{TOF}$ data available in EXFOR with this necessary information is considered as acceptable. The dissemination and preservation in EXFOR of more comprehensive datasets is obviously encouraged, and actually done on a case-by-case basis, depending on the end-use of the data and interactions with expert users such as evaluators.

Figure 1 and Table 2 illustrate the excellent data dissemination status of $\mathrm{n}_{-} \mathrm{TOF}$ data for fission. It shows the strong commitment of the fission team, which took the initiative early 2015 to make these data widely available to the nuclear data community.

Figure 2 shows the progress for the dissemination of capture data measured during Phase-I. The situation is not as satisfactory as for fission yet, essentially because capture measurements were performed by different teams, which partly turned to other activities along the different 


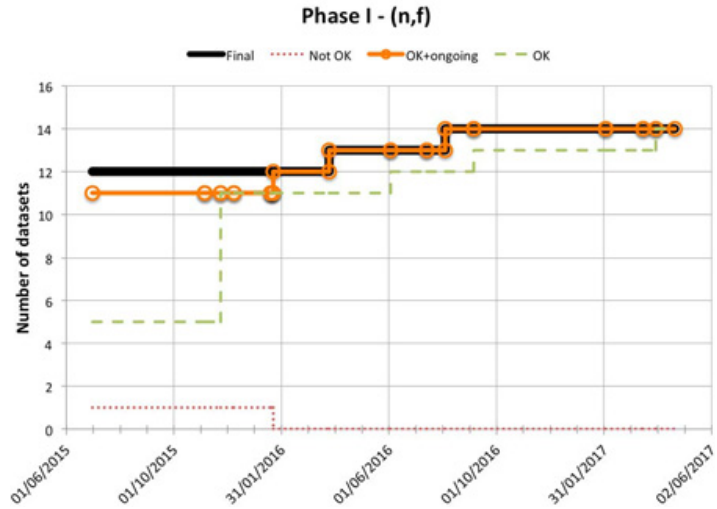

Figure 1. Evolution of data dissemination for Phase-I fission data. "Final" corresponds to datasets with a final publication; "OK" to datasets available in EXFOR with all necessary data; "OK + ongoing" includes also datasets under compilation by NRDC.

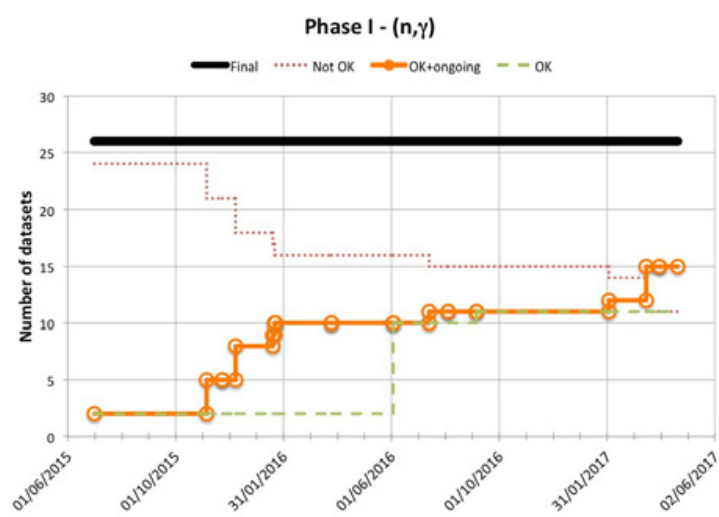

Figure 2. Evolution of data dissemination for $(\mathrm{n}, \gamma)$ data (Phase-I). See Fig. 1 for explanation of the legend.

n_TOF phases. Nevertheless, the situation is improving and contacts have been established with authors in order to retrieve old data and complement current EXFOR entries.

Table 2 shows the global dissemination status of $\mathrm{n}_{-} \mathrm{TOF}$ data, as of March 2017. Overall, 75\% of the datasets with a final publication are available in EXFOR with an acceptable status, i.e., all necessary data available. The current objective is to reach dissemination values higher than $80 \%$ for all reactions and phases. This should be possible thanks to ongoing efforts to retrieve as much as possible Phase-I capture yields from backups.

\section{Outlook}

Experimental data are useful and unique, accordingly they must be usable, actually used, and preserved. In order to improve the usability and long-term preservation of
Table 2. Dissemination status of n_TOF data as of March 2017.

\begin{tabular}{|c|c|c|c|}
\hline $\begin{array}{l}\text { n_TOF } \\
\text { phases }\end{array}$ & Reaction & $\begin{array}{c}\text { Datasets with } \\
\text { a final } \\
\text { publication }\end{array}$ & $\begin{array}{c}\text { Data } \\
\text { dissemination } \\
\text { status* }\end{array}$ \\
\hline \multirow{2}{*}{$\begin{array}{c}\text { Phase-I } \\
(\mathbf{2 0 0 1 - 2 0 0 4 )})\end{array}$} & $\mathbf{( n , \boldsymbol { \gamma } )}$ & 26 & $58 \%$ \\
\cline { 2 - 4 } & $\mathbf{( n , f )}$ & 14 & $100 \%$ \\
\hline \multirow{2}{*}{$\begin{array}{c}\text { Phase-II } \\
(\mathbf{2 0 0 9}-\mathbf{2 0 1 2})\end{array}$} & $(\mathbf{n}, \boldsymbol{\gamma})$ & 7 & $71 \%$ \\
\cline { 2 - 4 } & $(\mathbf{n , f})$ & 2 & $100 \%$ \\
\cline { 2 - 4 } & $\mathbf{A l l})$ & 2 & $100 \%$ \\
\hline $\begin{array}{c}\text { Phase-III } \\
(\mathbf{2 0 1 4}-. . .)\end{array}$ & $(\mathbf{n}, \mathbf{c p})$ & 1 & $\mathbf{8 2 \%}$ \\
\hline
\end{tabular}

*Percentage of final datasets with all necessary data already available in EXFOR or under compilation by NRDC.

n_TOF outcomes, the data dissemination is continuously monitored: new data are systematically released after the final publication, whereas the backlog of final data not available is being reduced. This is made possible thanks to an improved organisation for the data dissemination, and of course to the involvement of the whole $\mathrm{n}_{-} \mathrm{TOF}$ Collaboration, especially for the retrieval of old datasets. A list of measurements since 2001 is available on the n_TOF public TWiki webpage [13]. That list includes detailed information on the measured reactions: n_TOF phase, experimental area, detectors, energy range, data status (preliminary or final), main publications and corresponding EXFOR entries.

\section{References}

[1] The $\mathrm{n}_{-}$TOF Collaboration, CERN/INTC-O-011, INTC-2002-037, CERN-SL-2002-053 ECT (2003)

[2] N. Otuka, et al., Nucl. Data Sheets 120, 272 (2014)

[3] C. Guerrero, et al., Eur. Phys. J. A 49, 27 (2013)

[4] C. Weiß, et al., Nucl. Instr. Meth. A 799, 90 (2015)

[5] C. Guerrero, et al., Nucl. Instr. Meth. A 608, 424 (2009)

[6] M. Calviani, et al., Nucl. Instr. Meth. A 594, 220 (2008)

[7] C. Paradela, et al., Phys. Rev. C 82, 034601 (2010)

[8] D. Tarrío, et al., Phys. Rev. C 83, 044620 (2011)

[9] F. Gunsing, et al., Eur. Phys. J. Plus 131, 371 (2016)

[10] D. Tarrío, et al., Nucl. Instr. Meth. A 743, 79 (2014)

[11] F. Gunsing, et al., this conference I107 (2016)

[12] F. Gunsing, P. Schillebeeckx, V. Semkova, Report INDC(NDS)-0647 (IAEA, Vienna, 2013)

[13] http://twiki.cern.ch/NTOFPublic 